\title{
Errata: Automorphisms and Symmetries of Quantum Logics ${ }^{1}$
}

\author{
Věra Trnková ${ }^{2}$ \\ Received March 1, 1990
}

The following corrections were made in page proofs, but did not reach the publisher in time to appear in print. " $1211_{2}$ " means "p. 1211, line 2 from the bottom," and " $1211^{2 "}$ means "p. 1211, line 2 from the top."

\begin{tabular}{|c|c|c|}
\hline Page, line & Error & Correction \\
\hline $1196^{12}$ & $Q_{0}\left(L_{0}, M_{0}\right)$ & $Q_{0}=\left(L_{0}, M_{0}\right)$ \\
\hline $1197_{1}$ & atomist & atomistic \\
\hline $1198^{10}$ & $s_{a}(b)+s_{b}(s)$ & $s_{a}(b)+s_{b}(a)$ \\
\hline $1199^{5}$ & these & the \\
\hline $1200_{7}$ & $P_{g}$ & $P_{\mathscr{T}}$ \\
\hline $1200_{2}$ & $\{x, Y\}$ & $\{x, y\}$ \\
\hline $1202^{6}$ and $\mathrm{ff}$. & $\phi$ & $\varnothing$ \\
\hline $1202_{3}$ & $z \in B\{y\}, z \in B\{y\}$ & $z \in B\{x\}, z \in B\{y\}$ \\
\hline $1202_{1}$ & $B\{y\}$, and $\ldots$ & $B\{y\}, B\{x, y\}$, and $\ldots$ \\
\hline $\begin{array}{l}1204^{10} \\
1204^{17}\end{array}$ & covertices Section 2.1) & covertices (Section 2.1) \\
\hline $1205^{16}$ & $J=$ & $\Phi=$ \\
\hline $1205^{18}$ & $P^{+} \cup\left[m_{J}=m_{I} \mid I \in J\right]$ & $P^{+} \cup\left\{m_{J}\right\}=\left\{m_{I} \mid I \in \mathscr{J}\right\}$ \\
\hline $1206^{12}$ & $Q_{0}\left(L_{0}, M_{0}\right)$ & $Q_{0}=\left(L_{0}, M_{0}\right)$ \\
\hline $1206_{12}$ & set of atoms $A$ & set of atoms $\tilde{A}$ \\
\hline $1206_{11}$ & $\left.=s_{z}\right)_{t}=$ & $=\left(s_{z}\right)_{t}=$ \\
\hline $1206_{10}$ & $\left\{\tilde{s}_{a} \mid a \in A\right\}$ & $\left\{\tilde{s}_{a} \mid a \in \tilde{A}\right\}$ \\
\hline $1206_{4}$ & $\left\{s_{1} \mid a \in A\right\}$ & $\left\{s_{a} \mid a \in A\right\}$ \\
\hline
\end{tabular}

${ }^{1}$ This work appeared in the International Journal of Theoretical Physics, 28, 1195-1214 (1989).

${ }^{2}$ Mathematical Institute of the Charles University, 18600 Prague 8, Sokolovská 83, Czechoslovakia. 
Page, line

Error

Correction

\begin{tabular}{|c|c|c|}
\hline $1207_{11}$ & $\left\{G_{p} \uparrow p \in P\right\}$ & $\left\{G_{p} \mid p \in P\right\}$ \\
\hline $1207_{2}$ & Section 2. & Section 4.2 . \\
\hline $1208_{5}$ & $p=a_{a}$ & $p=s_{a}$ \\
\hline $1209^{12}$ & The & Then \\
\hline $1209_{15}$ & $Q_{0}\left(L_{0}, M_{0}\right)$ & $Q_{0}=\left(L_{0}, M_{0}\right)$ \\
\hline $1210^{1,6,13}$ & $p_{x, y}^{x, y}$ & $p_{x, y}^{x, y}$ \\
\hline $1210^{6}$ & $p_{x, y}^{x, y}$ & $p_{x, y}^{x, y}$ \\
\hline $1210^{7}$ and ff. & $\tilde{\phi}$ & $\tilde{\varnothing}$ \\
\hline $1210^{9}$ & $V_{p_{0}}$ & $V_{p}{ }^{\circ}$ \\
\hline $1210_{16,18}$ & $V_{p}^{0}$ & $V_{p}{ }^{0}$ \\
\hline $1210_{12,13}$ & $V_{p_{0}}$ & $V_{p}^{0}$ \\
\hline $1210_{1,5,9,17}$ & $\{x, y\}$ & $\{\overline{x, y}\}$ \\
\hline $1210_{1,5,9,15,16}$ & $\{\tilde{\boldsymbol{x}}\}$ & $\{\tilde{x}\}$ \\
\hline & $\{\tilde{y}\}$ & $\{\tilde{y}\}$ \\
\hline $1211^{1,2,3,4,6,11}$ & $\{\tilde{x}\}$ & $\{\tilde{x}\}$ \\
\hline & $\{\tilde{y}\}$ & $\{\tilde{y}\}$ \\
\hline $1211^{2,4,5,6,7,11}$ & $\{x, y\}$ & $\{\overline{x, y}\}$ \\
\hline $1211^{11}$ & $\{\tilde{y}\}$, & $\{\tilde{y}\}=\{\bar{y}\}$ \\
\hline $1211^{13}$ & $V_{p_{0}}$ & $V_{p}{ }^{0}$ \\
\hline $1211^{14}$ & $E_{p_{0}}$ & $E_{p}{ }^{0}$ \\
\hline $1211_{14}$ & $G_{p}\left(V_{p}, E_{p}\right)$ & $G_{p}=\left(V_{p}, E_{p}\right)$ \\
\hline $1211_{11}$ & $v: M \rightarrow M$ & $b: M \rightarrow M$ \\
\hline $1211_{2}$ & itself & $\begin{array}{l}\text { onto itself (and it is } \\
\text { identical on it) and } W \\
\text { also into itself. }\end{array}$ \\
\hline $1212^{4}$ & $V_{q} W$ & $V_{q} \cup W$ \\
\hline $1212_{14}$ & $\begin{array}{l}\text { every vertex ... no } \\
7 \text { cycle; }\end{array}$ & $\begin{array}{l}\text { and every vertex... } \\
\text { no } 7 \text { cycle, }\end{array}$ \\
\hline $1213_{3}$ & the unique point & the unique adjacent point \\
\hline $1214^{1}$ & show & shows \\
\hline $1214^{4}$ & $H_{1}\left(W, E_{1}\right)$ & $H_{1}=\left(N, E_{1}\right)$ \\
\hline
\end{tabular}

